\title{
Influence of Magnetic Field on Dielectric Breakdown in Transformer Oil Based Ferrofluids
}

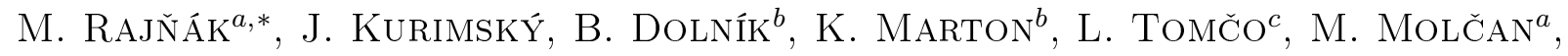 \\ P. KOPČANSKÝ ${ }^{a}, \mathrm{M}$. TIMKO ${ }^{a}$ \\ ${ }^{a}$ Institute of Experimental Physics SAS, Watsonova 47, 04001 Košice, Slovakia \\ ${ }^{b}$ Faculty of Electrotechnics and Informatics, Technical University, Letná 9/A, 04121 Košice, Slovakia \\ ${ }^{c}$ Faculty of Aviation, Technical University, Rampová 7, 04121 Košice, Slovakia
}

\begin{abstract}
In this paper the experimental study of the breakdown field strength in a transformer oil based ferrofluid is reported. The experiments are conducted on five ferrofluid samples with different magnetic volume fraction. The influence of external magnetic field on the breakdown field strength is investigated, when a quasi-homogenous magnetic field was applied in parallel and perpendicular configuration in regard to the electric field. The obtained results are analysed in accordance to the electron charging of ferrofluid nanoparticles theory.
\end{abstract}

DOI: $10.12693 /$ APhysPolA.126.248

PACS: 75.50.Mm, 77.22.Jp, 75.75.Jn

\section{Introduction}

Investigations on dielectric ferrofluids (magnetic fluids) are in great interest due to their potential use as a cooling and insulating medium in high power transformers. The finding of increased transformer oil breakdown strength with the addition of magnetite nanoparticles [1] led to extensive research work on dielectric properties of transformer oil based ferrofluids [2-4]. The presence of magnetic nanoparticles results in increased thermal conductivity, as well. Moreover, the transformer's leakage magnetic field and the produced heat can induce the thermomagnetic convection [5]. The research also showed the magnetodielectric anisotropy effect when the external magnetic field was applied to the investigated ferrofluids [2].

It was also found, that the propagation velocity of positive streamers are slower in ferrofluids than in the pure oil [1]. One can therefore estimate that the presence of magnetic nanoparticles in transformer oil inhibits the processes which lead to the dielectric breakdown. This paradoxical performance is due to the electron charging of nanoparticles. The charging process converts fast electrons from field ionization to slow negatively charged nanoparticle charge carriers with reduced effective mobility. This theoretical explanation was recently found by using numerical simulation methods [6].

In this study we confirm the increased breakdown field strength in the ferrofluid based on new transformer oil MOGUL TRAFO CZ-A (PARAMO). The influence of magnetic volume fraction in the ferrofluid and external magnetic field on the breakdown field strength will be analysed in regard to the charged magnetic nanoparticles model.

*corresponding author; e-mail: rajnak@saske.sk

\section{Ferrofluid characterization}

The investigated ferrofluid consists of the transformer oil in which magnetite nanoparticles covered with a layer of oleic acid molecules are dispersed. The magnetite $\left(\mathrm{Fe}_{3} \mathrm{O}_{4}\right)$ nanoparticles were synthesized from aqueous solution of $\mathrm{Fe}^{2+}$ and $\mathrm{Fe}^{3+}$ ions by co-precipitation method [7]. The narrow particle size distribution was found by fitting the AC magnetic susceptibility curve measured with IMEGO - DynoMag device. The calculated median particle diameter is $7.9 \mathrm{~nm}$ with the standard deviation of $2.4 \mathrm{~nm}$. The saturation magnetization of the originally prepared ferrofluid was $26.6 \mathrm{~A} \cdot \mathrm{m}^{2} \cdot \mathrm{kg}^{-1}$. The ferrofluid was further diluted to obtain 5 samples with different magnetic volume fraction $\Phi$ that was determined as

$$
\Phi=\frac{M_{s}}{M_{d}},
$$

where $M_{s}$ is the saturation magnetization of the ferrofluid sample and $M_{d}$ is the domain magnetization of the bulk magnetic particle $\left(M_{d}=446 \mathrm{kA} \cdot \mathrm{m}^{-1}\right.$ for magnetite $)$.

\section{Experimental details}

The breakdown voltage of all the samples was measured by High Voltage Oil Dielectric Tester DTS-60D with the accuracy less than $5 \%$. The separation distance $d$ between two electrodes of Rogowski shape was $0.2 \mathrm{~mm}$. The sample between the electrodes was exposed to AC voltage with the rate of rise of $500 \mathrm{~V} / \mathrm{s}$. The device is equipped with an automatic shutdown system starting after arc detection; therefore a minimal destruction of the tested sample was expected. The breakdown field strength was calculated simply as $E=U / d$, where $U$ is the breakdown voltage.

Firstly we measured the breakdown field strength in the pure oil sample by 15 independent measurements. Then, all the ferrofluid samples were investigated in the absence of external magnetic field as well as in the parallel 
and perpendicular configuration of electric $\boldsymbol{E}$ and magnetic field $\boldsymbol{B}$ (15 times). The quasi homogenous magnetic field of $120 \mathrm{mT}$ was generated by two plane permanent magnets attached to the measuring vessel. Finally, the average value and standard deviation for all measured samples were determined.

\section{Results and discussion}

The main results of this study are depicted in Fig. 1. The average values of the breakdown field strength are determined with the standard deviation of $3.06 \mathrm{MV} / \mathrm{m}$. In the case of zero magnetic field a clear increase in breakdown field strength is observed as the magnetic volume fraction increases. The breakdown field strength for the most concentrated ferrofluid sample $(\Phi=1.02 \%)$ is approximately 3 times higher than for the pure oil. Such behaviour was expected and it is in agreement with previous scientific results [1], [2], [4]. It might be explained by the assumption that the added nanoparticles act as electron scavengers, whereby the free electrons from the field ionization of the oil molecules are converted to slow negatively charged nanoparticles. Consequently, the speed of the streamers development is reduced.

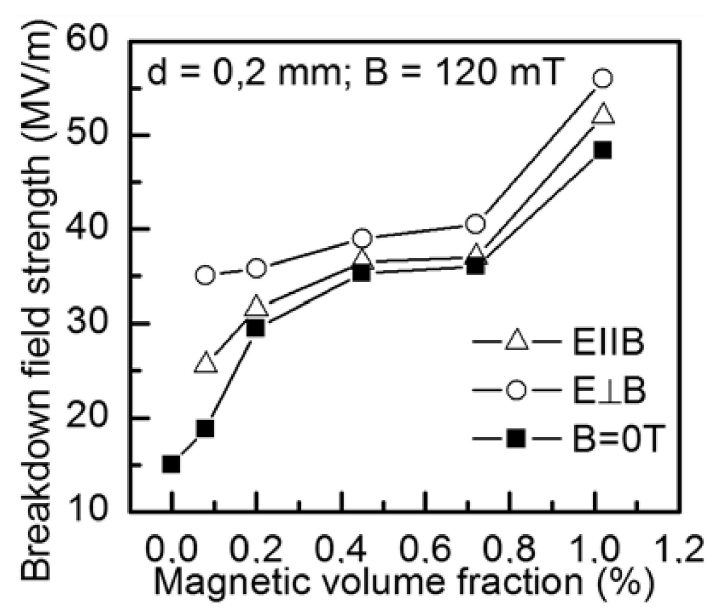

Fig. 1. The influence of magnetic field on the breakdown field strength of the transformer oil based ferrofluid as function of magnetic volume fraction in the oil.

The applied magnetic field induces a formation of chain like clusters of nanoparticles in the ferrofluid. When the particles in such clusters are negatively charged, they are still held together due to the magnetic field. In that case the motion of the clusters is even slower in comparison to the individual particles. This could be the reason of higher breakdown field strength values when the magnetic field is applied.

When the direction of magnetic field is parallel to the electric field, there is a zero angle between the motion of charged particles and the magnetic field direction. The Lorentz magnetic force is therefore zero and the motion is unaffected. In this parallel configuration the higher breakdown values are only due to the slow clusters and resulting slower speed of the streamers.

In the case of the perpendicular configuration the particles are trapped in clusters and they experience maximum Lorentz magnetic force. The motion of the charged particles describes a circular path as the Lorentz force is always perpendicular to the motion. This leads to helical motion of the charged clusters and finally leads to the slower streamer velocity and even higher breakdown field strength of the ferrofluid.

\section{Conclusion}

In the present study we considered the effect of external magnetic field on the transformer oil based feerrofluid breakdown field strength. The different values of breakdown field strength were found as a consequence of parallel and perpendicular orientation between magnetic and electric field. Besides the cluster formation an influence of the Lorentz force on the motion of the charged clusters is an additional cause of even higher breakdown field strength. However, a more precise experiment with a phase resolution of arc discharge initiation is required.

\section{Acknowledgments}

This work was supported by projects VEGA No. $2 / 0043 / 12,1 / 0045 / 12,1 / 0487 / 12$ and structural funds of EU No. 26220220061, 26220120046, 26220120055.

\section{References}

[1] V. Segal, A. Hjortsberg, A. Rabinovich, D. Nattrass, K. Raj, Conference Record of the 1998 IEEE International Symposium on Electrical Insulation, 2, 619 (1998).

[2] F. Herchl, K. Marton, L. Tomčo, P. Kopčanský, M. Timko, M. Koneracká, I. Kolcunová, J. Phys. Condens. Matter 20, 204110 (2008).

[3] M. Chiesa, S.K. Das, Colloids Surfaces Physicochem. Eng. Asp. 335, 88 (2009).

[4] J.-C. Lee, H.-S. Seo, Y.-J. Kim, Int. J. Therm. Sci. 62, 29 (2012).

[5] K. Raj, R. Moskowitz, US Patent, No. 5462685 (31 October 1995).

[6] J.G. Hwang, M. Zahn, F.M. O'Sullivan, L.A.A. Pettersson, O. Hjortstam, R. Liu, J. Appl. Phys. 107, 014310 (2010).

[7] L. Vekas, D. Bica, M.V. Avdeev, China Particuology, 5, 43 (2007). 